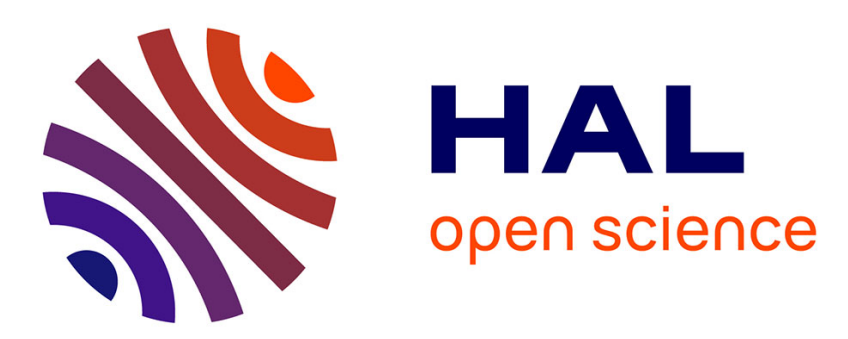

\title{
Stability analysis of uncertain sampled-data systems with incremental delay using looped-functionals
}

\author{
Alexandre Seuret, Corentin Briat
}

\section{To cite this version:}

Alexandre Seuret, Corentin Briat. Stability analysis of uncertain sampled-data systems with incremental delay using looped-functionals. Automatica, 2015, Automatica, 55 (5), pp.274-278. 10.1016/j.automatica.2015.03.015 . hal-01170887

\section{HAL Id: hal-01170887 \\ https://hal.science/hal-01170887}

Submitted on 2 Jul 2015

HAL is a multi-disciplinary open access archive for the deposit and dissemination of scientific research documents, whether they are published or not. The documents may come from teaching and research institutions in France or abroad, or from public or private research centers.
L'archive ouverte pluridisciplinaire HAL, est destinée au dépôt et à la diffusion de documents scientifiques de niveau recherche, publiés ou non, émanant des établissements d'enseignement et de recherche français ou étrangers, des laboratoires publics ou privés. 


\title{
Stability analysis of uncertain sampled-data systems with incremental delay using looped-functionals
}

\author{
Alexandre Seuret ${ }^{\mathrm{a}}$ and Corentin Briat ${ }^{\mathrm{b}}$ \\ ${ }^{\mathrm{a}}$ CNRS, LAAS, 7 avenue du colonel Roche, F-31400 Toulouse, France. \\ Univ. de Toulouse, LAAS, F-31400 Toulouse, France \\ ${ }^{\mathrm{b}}$ Department of Biosystems Science and Engineering (D-BSSE), \\ Swiss Federal Institute of Technology Zurich (ETH-Z), Switzerland
}

\begin{abstract}
The robust stability analysis of asynchronous and uncertain sampled-data systems with constant incremental input delay is addressed in the looped-functional framework. These functionals have been shown to be suitable for the analysis of impulsive systems as they allow one to express discrete-time stability conditions in an affine way, enabling then the consideration of uncertain and time-varying systems. The stability conditions are obtained by first reformulating the sampled-data system as an impulsive system, and by then considering a tailored looped-functional along with Wirtinger's inequality, a recently introduced inequality that has been shown to be less conservative than Jensen's inequality. Several examples are given for illustration.
\end{abstract}

Key words: Sampled-data systems, impulsive systems, looped-functionals; incremental delay, LMI.

\section{Introduction}

Sampled-data systems with periodic sampling have been extensively studied in the literature (Chen and Francis, 1995; Zhang et al., 2001) and this field is now very mature. Tools for designing robust controllers, which possibly optimize some performance criteria, are nowadays also well-established. In the case of asynchronous sampled-data system, however, these problems are still open. This is particularly important in the context of Networked Control Systems (Hespanha et al., 2007) where the presence of a network or, more generally the use of a shared resource, can corrupt the constancy of the sampling-period, making it time-varying, and the overall control system asynchronous. Several approaches to deal with stability analysis and/or control synthesis have been developed until now: discrete-time approaches (Suh, 2008; Heemels et al., 2010), inputdelay approaches (Mikheev et al., 1988; Teel et al., 1998; Fridman et al., 2004; Fridman, 2010; Liu and Fridman, 2012), robust analysis techniques (Kao and Fujioka, 2013), impulsive systems formulation (Sun et al., 1991;

Email addresses: aseuret@laas.fr (Alexandre Seuret), briatc@bsse.ethz.ch, corentin@briat.info (Corentin Briat).

URL: http://www . briat.info (Corentin Briat).

1 This work has been funded by the ANR under Grant LIMICOS ANR-12-BS03-005
Naghshtabrizi et al., 2008; Briat and Seuret, 2012), and the use of looped-functionals either considering directly the sampled-data system formulation (Seuret, 2012) or the impulsive system formulation (Briat and Seuret, 2012).

The problem addressed in this paper pertains on the robust stability analysis of uncertain and asynchronous sampled-data systems with input delay. A simpler instance of this problem has been studied in (Liu and Fridman, 2012) where only synchronous sampled-data systems are considered. The contribution of this paper is therefore twofold. The first one lies in the development of new stability conditions for linear impulsive systems with delays acting on the discrete-time part of the system. These stability conditions are obtained using a tailored looped-functional together with a new integral inequality introduced in (Seuret et al., 2013) in the context of time delay systems. The main differences with previous works on the topic, such as (Briat and Seuret, 2012)(Seuret et al., 2013), lie in 1) the use of a more advanced functional allowing us to cope with systems with time-varying matrices and; 2) the use of a new integral inequality that notably reduces the conservatism of the approach over the use of Jensen's inequality. By then relying on the equivalent reformulation of a sampleddata system into an impulsive system, the obtained stability conditions are applied to our specific problem, that is, the analysis of uncertain and asynchronous sampled-data systems with incremental delays. It is no- 
tably emphasized that the current approach improves over the method of (Liu and Fridman, 2012) in the case of synchronous samplings while being also able to deal with asynchrony. Examples are given for illustration.

Notations: For symmetric matrices $A, B, A-B \prec 0$ means that $A-B$ negative definite. The sets of symmetric and symmetric positive definite matrices of dimension $n$ are denoted by $\mathbb{S}^{n}$ and $\mathbb{S}_{+}^{n}$ respectively. The set of whole numbers is denoted by $\mathbb{N}$. Given a square matrix $A$, we define $\operatorname{Sym}[A]=A+A^{\top}$. For some vectors $\alpha, \beta$, the notation $\operatorname{col}\{\alpha, \beta\}$ denotes the vector obtained by stacking $\alpha$ and $\beta$ on the top of each others. Finally the notation $0_{m \times n}$ denotes the $m \times n$ zero-matrix.

\section{Problem formulation}

Let us consider linear systems of the form

$$
\begin{aligned}
& \dot{x}(t)=A_{0} x(t)+B_{0} u(t), t \geq 0, \\
& x(0)=x_{0},
\end{aligned}
$$

where $x, x_{0} \in \mathbb{R}^{n_{0}}$ and $u \in \mathbb{R}^{m}$ are the state of the system, the initial condition and the control input, respectively. The matrices $A_{0}$ and $B_{0}$ are not necessarily perfectly known but may be uncertain and/or time-varying. The control input $u$ obeys the following equation

$$
u(t)=K x\left(t_{k-d}\right), t \in\left[t_{k}, t_{k+1}\right), k \in \mathbb{N}
$$

where $K \in \mathbb{R}^{m \times n_{0}}$ is a controller gain and the sequence $\left\{t_{k}\right\}_{k \in \mathbb{N}}$ is the sequence of sampling instants. This sequence is assumed to be strictly increasing and does not admit any accumulation point, that is, we have that $t_{k} \rightarrow \infty$ as $k \rightarrow \infty$. We also make the additional assumption that the difference $T_{k}:=t_{k+1}-t_{k}$ belongs, for all $k \in \mathbb{N}$, to the interval $\left[T_{\min }, T_{\max }\right]$ where $T_{\min } \leq T_{\max }$. The incremental delay $d \in \mathbb{N}$ is assumed to be constant and known. The closed-loop system obtained from the interconnection of (1) and (2) is given, for all $k$ in $\mathbb{N}$, by

$$
\begin{aligned}
& \dot{x}(t)=A_{0} x(t)+B_{0} K x\left(t_{k-d}\right), t \in\left[t_{k}, t_{k+1}\right), \\
& x(\theta)=x_{0}, \theta \leq 0 .
\end{aligned}
$$

In the above model, the initial condition has been adapted in order to guarantee existence and uniqueness of solutions. Note that any other extension of the initial condition could have been done since stability of linear systems does not depend on initial conditions. When the sampling is periodic, i.e. $T_{k} \equiv T, k \in \mathbb{N}$, and the matrices $A_{0}$ and $B_{0}$ are known and constant, the system (3) can be easily analyzed using the LyapunovKrasovskii theorem or by augmenting the state vector; see e.g. (Hetel et al., 2008). The case of aperiodic sampling and uncertain matrices is, however, more difficult since discrete-time methods do not extend smoothly to this kind of systems, mainly due to the presence of exponential terms of the form $e^{A_{0} T}$ in the LMI conditions; see the extensive discussions in (Briat and Seuret, 2012). Several approaches have been developed to overcome these difficulties. The input-delay approach first introduced in (Teel et al., 1998) for nonlinear systems consists of rewriting the sampled state $x\left(t_{k-d}\right)$ as a delayed term of the form $x\left(t-\tau_{d}(t)\right)$ with sawtooth delay $\tau_{d}(t)$. The original sampled-data system is, in this case, transformed into a time-delay system. In this respect, the paper (Fridman et al., 2004) addresses the analysis of such systems using Lyapunov-Krasovskii functionals. Most of these previous approaches consider the particular case where the incremental delay $d$ is equal to 0 . When a computational delay indeed affects the computation of the control law, the analysis turns out to be more complex.

A distinct approach from the delay-based ones relies on the equivalent formulation of sampled-data systems as impulsive systems (Sun et al., 1991). Recently, hybrid techniques based on Lyapunov functionals have been developed to these systems (Naghshtabrizi et al., 2008; Goebel et al., 2009), yielding a second breath to this formulation. A linear impulsive system is defined by

$$
\dot{y}(t)=A y(t), \quad t \neq t_{k}, \quad y\left(t_{k}^{+}\right)=J y\left(t_{k}\right), \quad t=t_{k},
$$

for some state $y$ and with matrices $A, J \in \mathbb{R}^{n \times n}$. The sequence $\left\{t_{k}\right\}_{k \in \mathbb{N}}$ is assumed to be the same as for the sampled-data system (3). Above, the notation $y\left(t_{k}^{+}\right)$is the right-limit of $y(s)$ at $s=t_{k}$, i.e. $y\left(t_{k}^{+}\right):=\lim _{s \downarrow t_{k}} y(s)$.

In this framework, the system (3) can represented as (4) with $y(t):=\operatorname{col}\left\{x(t), x\left(t_{k}\right), \ldots, x\left(t_{k-d)}\right\}\right.$,

$$
A=\left[\begin{array}{cc}
A_{0} & B_{0} \bar{K} \\
0_{d n_{0} \times n_{0}} & 0_{d n_{0}}
\end{array}\right], J:=\left[\begin{array}{ccc}
{\left[I_{n_{0}}\right.} & 0_{n_{0} \times n_{0}(d-1)} & 0_{n_{0}} \\
& I_{d n_{0}} & 0_{d n_{0} \times n_{0}}
\end{array}\right],
$$

where $\bar{K}:=\left[\begin{array}{llll}0 & \ldots & 0 & K\end{array}\right]$. The interest of this formulation lies in the fact that the delayed sampled term is embedded in the state of the impulsive system and there is basically no distinction between the fact that we have one or more past terms in the control law. Indeed if the control law is affected by several delays, i.e. $u(t)=\sum_{i=0}^{d} K_{i} x\left(t_{k-i}\right)$, the resulting impulsive model can be simply rewritten by considering $\bar{K}:=\left[\begin{array}{llll}K_{0} & K_{1} & \ldots & K_{d}\end{array}\right]$. Of course the dimension of the impulsive system grows linearly according to the size of the delay $d$. Stated as such, there seems to be no striking difference between a standard discrete-time approach and an impulsive approach, and we may question the benefits of using the latter over the former. The main difference actually lies in the class of tools that will be used, i.e. looped-functionals, that will allow us to express a discrete-time stability condition in terms of continuoustime data $A$ and $J$ in a convex way, facilitating then the analysis of aperiodic sampled-data systems in the uncertain and time-varying case. The looped-functional based approach relies on the characterization of the trajectories of system (4) in a lifted domain (Yamamoto, 
1990; Briat and Seuret, 2012). Therefore, we view the entire state-trajectory as a sequence of functions

$$
\chi_{k}(\tau):=y\left(t_{k}+\tau\right) \text { with } \chi_{k}(0)=\lim _{s \downarrow t_{k}} y(s) .
$$

Looped-functionals then consider this definition of state for assessing stability in a novel manner. Notably, the positivity requirement of the functional can be shown to be relaxed.

\section{Stability analysis of linear impulsive systems}

\subsection{Main Result}

We have the following result:

Theorem 3.1 The impulsive system (4) with $T_{k}:=$ $t_{k+1}-t_{k} \in\left[T_{\min }, T_{\max }\right], k \in \mathbb{N}$, is asymptotically stable if there exist matrices $P, Z \in \mathbb{S}_{+}^{n}, Q, S, X \in \mathbb{S}^{n}$, $R, U \in \mathbb{R}^{n \times n}$ and $Y_{1}, Y_{2} \in \mathbb{R}^{n \times 3 n}$ such that the LMIs

$$
\Psi(\theta) \prec 0 \text { and } \Phi(\theta) \prec 0
$$

hold for all $\theta \in\left\{T_{\min }, T_{\max }\right\}$ where

$$
\begin{aligned}
\Psi(\theta) & :=F_{0}(\theta)+\theta\left(F_{2}+F_{3}\right), \\
\Phi(\theta): & =\left[\begin{array}{ccc}
F_{0}(\theta)+\theta\left(F_{1}-F_{3}\right) & \theta Y_{1}^{\top} & \theta Y_{2}^{\top} \\
\star & -\theta Z & 0 \\
\star & \star & -\frac{\theta}{3} Z
\end{array}\right],
\end{aligned}
$$

with $M_{y}=\left[\begin{array}{lll}I & 0 & 0\end{array}\right], M_{\zeta}=\left[\begin{array}{lll}I & -J & 0\end{array}\right], M_{\nu}=\left[\begin{array}{lll}I & J & -2 I\end{array}\right]$, $M_{-}=\left[\begin{array}{lll}0 & I & 0\end{array}\right], M_{+}=\left[\begin{array}{lll}0 & 0 & I\end{array}\right], F_{1}=-\operatorname{Sym}\left[M_{+} U J M_{-}\right]$, $F_{3}=M_{-}^{\top} J^{\top} X J M_{-}$and

$$
\begin{aligned}
F_{0}(\theta)= & F_{00}(\theta)-\operatorname{Sym}\left[Y_{1}^{\top} M_{\zeta}+3 Y_{2}^{\top} M_{\nu}\right], \\
F_{00}(\theta)= & \theta M_{y}^{\top}\left(A^{\top} P+P A\right) M_{y}-M_{\zeta}^{\top} Q M_{\zeta}-\theta M_{+}^{\top} S M_{+} \\
& +M_{-}^{\top}\left(J^{\top} P J-P\right) M_{-}-\operatorname{Sym}\left[M_{\zeta}^{\top} R M_{y}\right], \\
F_{2}= & \operatorname{Sym}\left[M_{y}^{\top} A^{\top} Q M_{\zeta}+M_{y}^{\top} A^{\top} R M_{y}+M_{\zeta}^{\top} R A M_{y}\right. \\
& \left.+M_{+}^{\top} S M_{y}\right]+M_{y}^{\top} U J M_{-}+M_{y}^{\top} A^{\top} Z A M_{y} .
\end{aligned}
$$

When the above conditions are satisfied, then we have that $J^{\top} e^{A^{\top} \theta} P e^{A \theta} J-P \prec 0$ holds for all $\theta \in\left[T_{\text {min }}, T_{\text {max }}\right]$ and, therefore, the quadratic form $V(y)=y^{\top} P y$ is a Lyapunov function for the aperiodic impulsive system $y\left(t_{k+1}\right)=e^{A T_{k}} J y\left(t_{k}\right)$ with $T_{k} \in\left[T_{\min }, T_{\max }\right]$.

Proof : First we recall the Wirtinger-based integral inequality from (Seuret et al., 2013).
Lemma 3.2 Let $\omega:[a, b] \rightarrow \mathbb{R}^{n}$ be a differentiable function over $(a, b)$ having square integrable first order derivative. Then, for all $Z \in \mathbb{S}_{+}^{n}$, we have

$$
\int_{a}^{b} \dot{\omega}(u)^{\top} Z \dot{\omega}(u) d u \geq \frac{1}{b-a} \Omega_{1}^{\top} Z \Omega_{1}+\frac{3}{b-a} \Omega_{2}^{\top} Z \Omega_{2},
$$

where $\Omega_{1}=\omega(b)-\omega(a)$ and $\Omega_{2}=\omega(b)+\omega(a)-2 /(b-$ a) $\int_{a}^{b} \omega(u) d u$.

Following Theorem 2.4 from (Briat and Seuret, 2012), let us consider the functional $\mathcal{W}_{k}$ as

$\mathcal{W}_{k}\left(\tau, \chi_{k}, \chi_{k-1}\right):=\tau \Lambda_{k}+T_{k}\left[V\left(\chi_{k}(\tau)\right)+\mathcal{V}\left(\tau, \chi_{k}, T_{k}\right)\right]$

where $\Lambda_{k}:=V\left(\chi_{k}(0)\right)-V\left(\chi_{k-1}\left(T_{k-1}\right)\right), V(y)=y^{\top} P y$ and $\mathcal{V}$ such that

$$
\begin{aligned}
T_{k} \mathcal{V}\left(\tau, \chi_{k}, T_{k}\right) & =\left(T_{k}-\tau\right) \zeta_{k}(\tau)^{\top}\left[Q \zeta_{k}(\tau)+2 R \chi_{k}(\tau)\right] \\
& +\left(T_{k}-\tau\right) \tau \nu_{k}(\tau)^{\top}\left[S \nu_{k}(\tau)+2 U J \chi_{k}(0)\right] \\
& +\left(T_{k}-\tau\right) \tau \chi_{k}(0)^{\top} J^{\top} X J \chi_{k}(0) \\
& +\left(T_{k}-\tau\right) \int_{0}^{\tau} \dot{\chi}_{k}(s)^{\top} Z \dot{\chi}_{k}(s) d s
\end{aligned}
$$

where $\zeta_{k}(\tau)=\chi_{k}(\tau)-\chi_{k}(0), \dot{\chi}_{k}(\tau)=A \chi_{k}(\tau)$, $\nu(\tau)=\frac{1}{\tau} \int_{0}^{\tau} \chi_{k}(s) \mathrm{d} s, P, Z \in \mathbb{S}_{+}^{n}, Q, S, X \in \mathbb{S}^{n}$ and $U, R \in \mathbb{R}^{n \times n}$. It is important to point out that the matrices $Q, R, S$ and $U$ are indefinite. Since $\mathcal{V}\left(0, z, T_{k}\right)=\mathcal{V}\left(T_{k}, z, T_{k}\right)=0$ for all $z \in C\left(\left[0, T_{k}\right], \mathbb{R}^{n}\right)$ and for all $T_{k} \in\left[T_{\min }, T_{\max }\right]$, Theorem 2.4 from (Briat and Seuret, 2012) can be applied to get that

$$
\begin{aligned}
\dot{\mathcal{W}}_{k}= & T_{k} \frac{d}{d \tau}\left[V\left(\chi_{k}(\tau)\right)+\mathcal{V}\left(\tau, \chi_{k}, T_{k}\right)\right]+\Lambda_{k} \\
= & \xi_{k}(\tau)^{\top}\left[F_{00}\left(T_{k}\right)+\tau F_{1}+\left(T_{k}-\tau\right) F_{2}\right. \\
& \left.+\left(T_{k}-2 \tau\right) F_{3}\right] \xi_{k}(\tau)-\int_{0}^{\tau} \dot{\chi}_{k}(s)^{\top} Z_{\dot{\chi}_{k}}(s) \mathrm{d} s
\end{aligned}
$$

where $F_{00}\left(T_{k}\right), \quad F_{2}$ and $F_{3}$ are given in (9) and $\xi_{k}(\tau):=\operatorname{col}\left\{\chi_{k}(\tau), \chi_{k-1}\left(T_{k-1}\right), \nu_{k}(\tau)\right\}$. Noting that $\chi_{k}(0)=J \chi_{k-1}\left(T_{k-1}\right)$, Lemma 3.2 yields the inequality

$$
\begin{aligned}
-\int_{0}^{\tau} \dot{\chi}_{k}(s)^{\top} Z \dot{\chi}_{k}(s) \mathrm{d} s \leq \\
\quad-(1 / \tau) \xi_{k}(\tau)^{\top}\left(M_{\zeta}^{\top} Z M_{\zeta}+3 M_{\nu}^{\top} Z M_{\nu}\right) \xi_{k}(\tau)
\end{aligned}
$$

Following (Briat, 2011), both computational tractability and accuracy are improved by turned the RHS into an affine expression of $\tau$. This can be performed by using the bounds discussed in the same paper to get

$$
\begin{aligned}
-(1 / \tau) \xi_{k}(\tau)^{\top} & \left(M_{\zeta}^{\top} Z M_{\zeta}+3 M_{\nu}^{\top} Z M_{\nu}\right) \xi_{k}(\tau) \leq \\
-\xi_{k}(\tau)^{\top} & \left(\operatorname{Sym}\left[Y_{1} M_{\zeta}+3 Y_{2} M_{\nu}\right]\right. \\
& \left.-\tau\left(Y_{1}^{\top} Z^{-1} Y_{1}-3 Y_{2}^{\top} Z^{-1} Y_{2}\right)\right) \xi_{k}(\tau)
\end{aligned}
$$


Table 1

Allowable interimpulse intervals for Example 1.

\begin{tabular}{|c|l|l|}
\hline Methods & Periodic & Aperiodic \\
\hline Briat et al. $(2012)$ & {$[0.1824,0.5760]$} & {$[0.1907,0.5063]$} \\
Theorem 3.1 & {$[0.1824,0.5774]$} & {$[0.1824,0.5766]$} \\
\hline
\end{tabular}

Combining the previous statements all together, we can state that the system (4) with $T$-periodic impulses is asymptotically stable if $\dot{\mathcal{W}}_{k}$ is negative definite over $\tau \in$ $[0, T]$. A sufficient condition of asymptotic stability is then that the parameter-dependent LMI

$$
F_{0}(T)+\tau \bar{F}_{1}+(T-\tau) F_{2}+(T-2 \tau) F_{3} \prec 0,
$$

holds for all $\tau \in[0, T]$ where $\bar{F}_{1}=F_{1}+Y_{1}^{\top} Z^{-1} Y_{1}+$ $3 Y_{2}^{\top} Z^{-1} Y_{2}$. Since this LMI is affine in $\tau$ (hence convex), to check its negative definiteness over the entire interval $\left[0, T_{k}\right]$, it is necessary and sufficient to check it at the vertices of the set, that is only over the finite set $\tau \in\left\{0, T_{k}\right\}$. A Schur complement on the quadratic terms $T_{k} Y_{1}^{\top} Z^{-1} Y_{1}$ and $3 T_{k} Y_{2}^{\top} Z^{-1} Y_{2}$ finally yields $\Phi\left(T_{k}\right) \prec 0$ and $\Psi\left(T_{k}\right) \prec 0$. Since $\Psi\left(T_{k}\right) \prec 0$ and $\Phi\left(T_{k}\right) \prec 0$ are affine in $T_{k} \in\left[T_{\min }, T_{\max }\right]$, we can apply the same arguments as above to show that we just need to check them at the values in the finite set $\left\{T_{\min }, T_{\max }\right\}$.

Finally, if the LMI condition (7) are satisfied for all $\theta \in\left[T_{\min }, T_{\max }\right]$, this implies that $\dot{\mathcal{W}}<0$, which, according to Theorem 4.2 from (Briat and Seuret, 2012), ensures the asymptotic stability of the system (4).

\subsection{An example on impulsive systems}

Example 1 (Briat and Seuret, 2012) Let us consider the system (4) with matrices

$$
A=\left[\begin{array}{cc}
-1 & 0.1 \\
0 & 1.2
\end{array}\right], \quad J=\left[\begin{array}{cc}
1.2 & 0 \\
0 & 0.5
\end{array}\right]
$$

Note that, the continuous-time dynamics of the first state is stable while the second is unstable. Conversely, the matrix $J$ has a stable eigenvalue for the second state and an unstable one for the first state. It is hence expected that the range of admissible inter-impulse distances is a connected interval excluding 0 and $+\infty$. An eigenvalue analysis gives the admissible range [0.1824, 0.5776] of inter-impulse periods. Table 1 presents the results. It is sowed that the Theorem 3.1 precises the condition from (Briat and Seuret, 2012). The improvements for the periodic and the aperiodic cases are due to the use of the integral inequality Lemma 3.2.

\subsection{Stability of delayed sampled-data systems}

In the following, we will consider more specifically the case of sampled-data systems affected by an incremental delay $d$. Therefore, from now on, the matrices $A$ and $J$ are given by those in (5). We also denote by $n$ the actual dimension of the state of the impulsive system representing the sampled-data system, that is $n=n_{0}(d+1)$. An important difference with respect to Theorem 3.1, is that the particular structure of the matrices $A$ and $J$ are exploited in order to reduce the number of variables in the LMI conditions and lower the computational complexity of the approach.

Let us assume now that the sampled-data system (1) is uncertain and subject to time-varying uncertainties, i.e.

$$
\left[\begin{array}{ll}
A_{0} & B_{0}
\end{array}\right] \in \mathcal{C} o\left(\left[\begin{array}{ll}
A_{0}^{1} & B_{0}^{1}
\end{array}\right], \ldots,\left[\begin{array}{ll}
A_{0}^{M} & B_{0}^{M}
\end{array}\right]\right),
$$

where $\mathcal{C}$ o denotes the convex hull operator. Based on the impulsive system formulation, we are in position to provide the main result on the robust stability of aperiodic sampled-data systems:

Theorem 3.3 The uncertain aperiodic sampled-data system (3)-(15) with $T_{k} \in\left[T_{\min }, T_{\max }\right], k \in \mathbb{N}$, is asymptotically stable if there exist matrices $P \in \mathbb{S}_{+}^{n}, \tilde{Z} \in \mathbb{S}_{+}^{n_{0}}$, $\tilde{Q}, \tilde{S}, \in \mathbb{S}^{n_{0}}, X \in \mathbb{S}^{n}, \tilde{R}, \tilde{U} \in \mathbb{R}^{n_{0} \times n}$ and $\tilde{Y}_{1}, \tilde{Y}_{2} \in$ $\mathbb{R}^{n_{0} \times\left(n+2 n_{0}\right)}$ such that the LMIs $\tilde{\Psi}\left(\theta, A_{0}^{j}, B_{0}^{j}, K\right) \prec 0$ and $\tilde{\Phi}\left(\theta, A_{0}^{j}, B_{0}^{j}, K\right) \prec 0$ hold for all $\theta \in\left\{T_{\min } T_{\max }\right\}$ and all $j=1, \ldots, M$ where for all matrices $A_{0}, B_{0}, K$ of appropriate dimension,

$$
\begin{aligned}
& \tilde{\Psi}\left(\theta, A_{0}, B_{0}, K\right):=G_{0}(\theta)+\theta\left(G_{2}+G_{3}\right) \\
& \tilde{\Phi}\left(\theta, A_{0}, B_{0}, K\right):=\left[\begin{array}{ccc}
G_{0}(\theta)+\theta\left(G_{1}-G_{3}\right) & \theta \tilde{Y}_{1}^{T} & \theta \tilde{Y}_{2}^{\top} \\
\star & -\theta \tilde{Z} & 0 \\
\star & \star & -\frac{\theta}{3} \tilde{Z}
\end{array}\right] \\
& G_{0}(\theta)=G_{00}(\theta)-\operatorname{Sym}\left[\tilde{Y}_{1}^{\top} N_{\zeta}+3 \tilde{Y}_{2}^{\top} N_{\nu}\right], \\
& G_{00}(\theta)=\theta N_{y}^{\top}\left(A^{\top} P+P A\right) N_{y}-N_{\zeta}^{\top} \tilde{Q} N_{\zeta}-\theta N_{+}^{\top} S N_{+} \\
& +N_{-}^{\top}\left(J^{\top} P J-P\right) N_{-}-\operatorname{Sym}\left[N_{\zeta}^{\top} \tilde{R} N_{y}\right] \text {, } \\
& G_{1}=-\operatorname{Sym}\left[N_{+} U N_{-}\right], \quad G_{3}=N_{-}^{\top} J^{\top} X J N_{-}, \\
& G_{2}=\operatorname{Sym}\left[N_{y}^{\top} \tilde{A}^{\top} \tilde{Q} N_{\zeta}+N_{y}^{\top} \tilde{A}^{\top} \tilde{R} N_{y}+N_{\zeta}^{\top} \tilde{R} A N_{y}\right. \\
& \left.+N_{+}^{\top} \tilde{S} J N_{y}+N_{y}^{\top} \tilde{U} J N_{-}\right]+N_{y}^{\top} \tilde{A} \tilde{Z}^{\top} \tilde{Z} \tilde{A} N_{y},
\end{aligned}
$$

and $\tilde{A}=\left[\begin{array}{ll}A_{0} & B \bar{K}\end{array}\right], N_{\zeta}=\left[\begin{array}{lll}I_{n_{0}} & -I_{n_{0}} & 0_{n_{0} \times n}\end{array}\right], N_{\nu}=$

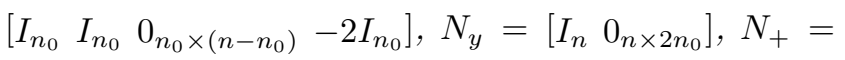
$\left[\begin{array}{ll}0_{n_{0} \times\left(n+n_{0}\right)} & I_{n_{0}}\end{array}\right], N_{-}=\left[\begin{array}{lll}0_{n \times n_{0}} & I_{n} & 0_{n \times n_{0}}\end{array}\right]$.

Proof: Due to space limitations, the proof is only sketched. When considering the uncertain sampleddata system (3)-(15), the corresponding impulsive system (4)-(5) has redundant information in its formulation. It is, indeed, possible to show that there exists a matrix $W$ of appropriate dimensions such that $\xi_{k}(\tau)=W\left[\rho_{k}(\tau)^{\top} X_{k}^{\top} x\left(t_{k-N}\right)^{\top} v_{k}(\tau)\right]^{\top}$, where $\rho_{k}(\tau)$ and $v_{k}(\tau)$ contain the first $n_{0}$ entries of $\chi_{k}(\tau)$ and $\nu_{k}(\tau)$, respectively. The vector $X_{k}$ is defined, as before, as 
$X_{k}=\operatorname{col}\left\{x\left(t_{k}\right), \ldots, x\left(t_{k-N+1}\right)\right\}$. The proof then consists of applying first Finsler's lemma (Skelton et al., 1997) and then reducing the number of variables by exploiting the particular structure of the matrices of the system. Noting finally that the matrices $\tilde{\Psi}$ and $\tilde{\Phi}$ are convex in their second and third arguments allows us to extend the conditions to uncertain sampled-data systems with polytopic uncertainties.

Remark 1 The conditions of Theorem 3.3 deals with aperiodic samplings. It is however possible to address the problem of periodic samplings by selecting $T_{\min }=T_{\max }$.

Remark 2 The numerical burden associated with the conditions of Theorem 3.3 increases exponentially with the value of the incremental delay d. In Seuret et al. (2014), a mixed continuous/discrete-time analysis is performed thanks to a Lyapunov-Krasovskii functional to deal with the discrete-time-delay system.

\subsection{Examples on sampled-data systems}

Example 2 (Zhang et al. (2001)) Let us consider the sampled-data system (3) with matrices

$$
A_{0}=\left[\begin{array}{cc}
0 & 1 \\
0 & -0.1
\end{array}\right], \quad B_{0}=\left[\begin{array}{c}
0 \\
-0.1
\end{array}\right], \quad K=[3.75,11.5]
$$

An eigenvalue-based analysis shows that this sampleddata system is asymptotically stable for any constant sampling period smaller than $T_{\max }=1.7294,0.7637$, 0.4638 when $d=0,1,2$, respectively.

Periodic sampling case: When $d=0$, (Liu and Fridman, 2012) show that stability of the sampled-data system is preserved for any constant sampling-period in the intervals $(0,1.39]$. In (Briat and Seuret, 2012), the interval $(0,1.7239]$ is found while Theorem 3.3 (with $T_{\text {min }}=$ $\left.T_{\max }=T\right)$ yields $(0,1.7294]$ showing then exactness of the estimate for this example. When $d=1,2$, Theorem 4.1 returns 0.7637 and 0.4638 , respectively. This example demonstrates that, even if the proposed method is conservative in general, the conservatism can be vanishingly small for some systems.

Aperiodic sampling case: When $d=0$, the conditions from (Liu and Fridman, 2012) preserves stability for any sampling in the intervals $(0,1.39]$, The approach considered in (Naghshtabrizi et al., 2008) and (Fridman, 2010) indicate that stability is preserved for any aperiodic sampling in the intervals $(0 ; 1.113]$, and $(0 ; 1.69]$, respectively. The looped-functional approaches discussed in (Seuret, 2012; Briat and Seuret, 2012) yield the interval (0,1.7239]. Theorem 3.3 delivers the interval (0, 1.7293]. The conditions yield tighter estimates of the interval. When $d=1,2$, Theorem 3.3 ensures stability for all samplings that belongs to $\left[10^{-3}, 0.73\right]$ and $\left[10^{-3}, 0.43\right]$, respectively, which, again, illustrates the efficiency of the proposed method.
Table 2

Intervals of allowable aperiodic samplings for Example 3.

\begin{tabular}{|c|l|}
\hline Methods $(d=0)$ & Sampling intervals \\
\hline \hline (Seuret, 2012) & {$\left[\begin{array}{ll}0.4 & 1.25\end{array}\right],\left[\begin{array}{ll}1.2 & 1.57\end{array}\right]$} \\
(Seuret et al., 2013) & {$\left[\begin{array}{ll}0.4 & 1.31\end{array}\right],\left[\begin{array}{ll}0.8 & 1.56\end{array}\right]$} \\
(Briat and Seuret, 2012) & {$\left[\begin{array}{lll}0.4 & 1.43\end{array}\right],\left[\begin{array}{ll}0.8 & 1.58\end{array}\right]$} \\
(Kao and Fujioka, 2013) & {$\left[\begin{array}{lll}0.4 & 1.39\end{array}\right],\left[\begin{array}{ll}0.8 & 1.61\end{array}\right]$} \\
& \\
Theorem 3.3 & {$\left[\begin{array}{ll}0.4 & 1.66\end{array}\right],\left[\begin{array}{ll}0.8 & 1.86\end{array}\right]$} \\
\hline
\end{tabular}

Table 3

Allowable sampling intervals for Example 4.

\begin{tabular}{|c|l|l|}
\hline Methods & Periodic & Aperiodic \\
\hline $\begin{array}{c}\text { (Liu and Fridman, 2012) } \\
\text { Theorem 3.3 }\end{array}$ & {$\left[10^{-5} 0.499\right]$} & $\emptyset$ \\
\hline $\left.10^{-5} 0.788\right]$ & {$[0.3,0.645]$} \\
\hline
\end{tabular}

Example 3 (Gu et al. (2003)) Let us consider the sampled-data system (3) with $d=0$ and with matrices

$$
A_{0}=\left[\begin{array}{cc}
0 & 1 \\
-2 & 0.1
\end{array}\right], \quad B_{0}=\left[\begin{array}{l}
0 \\
1
\end{array}\right], \quad K=\left[\begin{array}{ll}
1 & 0
\end{array}\right]
$$

An eigenvalue-based analysis shows that this sampleddata system is asymptotically stable for any constant sampling period in the interval [0.2007,2.020]. First, the stability conditions from (Naghshtabrizi et al., 2008), (Fridman, 2010) fail assessing stability. According to Table 2, the condition from Theorem 3.3 improves again the ones form the literature on this example.

Example 4 (Liu and Fridman (2012)) Let us consider now the sampled-data system (3)

$\dot{x}(t)=\left[\begin{array}{cc}0 & 1 \\ g(t) & 0\end{array}\right] x(t)+\left[\begin{array}{l}0 \\ 1\end{array}\right] u(t)$
$u(t)=\left[\begin{array}{ll}-0.35 & 0\end{array}\right] x\left(t_{k}\right)+\left[\begin{array}{ll}0.1 & 0\end{array}\right] x\left(t_{k-3}\right), \forall t \in\left[t_{k}, t_{k+1}\right)$

and where the function $g$ is such that $|g(t)| \leq 0.1$, for all $t \geq 0$. Due to the time-varying nature of the function $g$, stability cannot be analyzed using the discrete-time stability result. For indication, a gridding approach combined with eigenvalue analysis (which is only valid in the timeinvariant case) yields that the maximal periodic sampling period for such system is 0.7996. The method of (Liu and Fridman, 2012) is not applicable for aperiodic samplings. Results are presented in Table 3 and shows that Theorem 3.3 delivers less conservative results on this example. Of course these improvement is obtained at the price of an additional complexity. Indeed the number of decision variables in (Liu and Fridman, 2012) is $12 n^{2}+6 n$ while in our approach, we have $36 n^{2}+5 n$. 


\section{Conclusion}

A new looped-functional-based approach has been proposed for analyzing the stability of periodic and aperiodic uncertain sampled-data systems with incremental delays. The conditions have been obtained using Wirtinger's inequality along with a complexity reduction procedure. Several examples illustrate the efficiency of the approach over existing ones.

\section{References}

Briat, C., 2011. Convergence and equivalence results for the Jensen's inequality - application to time-delay and sampled-data systems. IEEE Trans. on Automatic Control 56(7), 1660-1665.

Briat, C., Seuret, A., 2012. A looped-functional approach for robust stability analysis of linear impulsive systems. Systems \& Control Letters 61, 980-988.

Chen, T., Francis, B., 1995. Optimal sampled-data control systems. Springer-Verlag, Berlin, Germany.

Fridman, E., 2010. A refined input delay approach to sampled-data control. Automatica 46 (2), 421-427.

Fridman, E., Seuret, A., Richard, J.-P., 2004. Robust sampled-data stabilization of linear systems: An input delay approach. Automatica 40 (8), 1141-1446.

Goebel, R., Sanfelice, R., Teel, A., 2009. Hybrid dynamical systems. Automatica 29 (2), 28-93.

Gu, K., Kharitonov, V.-L., Chen, J., 2003. Stability of time-delay systems. Birkhauser.

Heemels, W. H., Teel, A. R., van de Wouw, N., Nesic, D., 2010. Networked control systems with communication constraints: Tradeoffs between transmission intervals, delays and performance. IEEE Trans. on Automatic Control 55 (8), 1781-1796.

Hespanha, J., Naghshtabrizi, P., Xu, Y., 2007. A survey of recent results in networked control systems. Proceedings of the IEEE 95 (1), 138-162.

Hetel, L., Daafouz, J., Iung, C., 2008. Equivalence between the Lyapunov-Krasovskii functionals approach for discrete delay systems and that of the stability conditions for switched systems. Nonlinear Analysis: Hybrid Systems 2 (3), 697-705.

Kao, C.-Y., Fujioka, H., 2013. On stability of systems with aperiodic sampling devices. IEEE Trans. on Automatic Control 58 (8), 2085-2090 .

Liu, K., Fridman, E., 2012. Wirtinger's inequality and Lyapunov-based sampled-data stabilization. Automatica 48 (1), 102-108.

Mikheev, Y.V., Sobolev, V.A., and Fridman, E., 1988. Asymptotic analysis of digital control systems. Autom. Remote Control 49(9), 1175-1180.

Naghshtabrizi, P., Hespanha, J., Teel, A., 2008. Exponential stability of impulsive systems with application to uncertain sampled-data systems. Systems \& Control Letters 57 (5), 378-385.

Seuret, A., 2012. A novel stability analysis of linear systems under asynchronous samplings. Automatica 48 (1), 177-182.
Seuret, A., Gouaisbaut, F., 2013. Wirtinger-based integral inequality: Application to time-delay systems. Automatica 49 (9), 2860-2866.

Seuret, A., Briat, C., Gouaisbaut, F., 2014. Stability analysis of asynchronous sampled-data systems with discrete-time constant input delay. Proceedings of the 53th IEEE Conference on Decision and Control (CDC14).

Skelton, R., Iwasaki, T., Grigoriadis, K., 1997. A Unified Algebraic Approach to Linear Control Design. Taylor \& Francis.

Suh, Y., 2008. Stability and stabilization of nonuniform sampling systems. Automatica 44 (12), 3222-3226.

Sun, W., Nagpal, K. M., Khargonekar, P. P., 1991. $H_{\infty}$ control and filtering with sampled measurements. In: American Control Conference, 1991. pp. 1652-1657.

Teel, A., Nesic, D., Kokotovic, P., 1998. A note on inputto-state stability of sampled-data nonlinear systems. In: IEEE Conference on Decision and Control. Vol. 3. pp. 2473-2478.

Yamamoto, Y., 1990. New approach to sampled-data control systems - a function space method. In: Proceedings of the 29th Conference on Decision and Control. pp. $1882-1887$.

Zhang, W., Branicky, M., Phillips, S., 2001. Stability of Networked Control Systems. IEEE Control Systems Magazine 21(1), 84-99. 